\title{
Influence of Population Immunosuppression and Past Vaccination on Smallpox Reemergence
}

\author{
C. Raina Maclntyre, Valentina Costantino, Xin Chen, Eva Segelov, Abrar Ahmad Chughtai, \\ Anthony Kelleher, Mohana Kunasekaran, John Michael Lane
}

We built a SEIR (susceptible, exposed, infected, recovered) model of smallpox transmission for New York, New York, USA, and Sydney, New South Wales, Australia, that accounted for age-specific population immunosuppression and residual vaccine immunity and conducted sensitivity analyses to estimate the effect these parameters might have on smallpox reemergence. At least 19\% of New York's and $17 \%$ of Sydney's population are immunosuppressed. The highest smallpox infection rates were in persons 0-19 years of age, but the highest death rates were in those $\geq 45$ years of age. Because of the low level of residual vaccine immunity, immunosuppression was more influential than vaccination on death and infection rates in our model. Despite widespread smallpox vaccination until 1980 in New York, smallpox outbreak severity appeared worse in New York than in Sydney. Immunosuppression is highly prevalent and should be considered in future smallpox outbreak models because excluding this factor probably underestimates death and infection rates.

A mallpox virus was eradicated in 1980 but remains a cat$\checkmark$ egory A bioterrorism agent (1). The only official stocks of the virus are in the United States and Russia (2), but unofficial stocks could be present elsewhere. Advances in synthetic biology of poxviruses and availability of the full variola genome sequence make synthesis of smallpox virus in the laboratory possible (3). Smallpox could reemerge as a result of bioterrorism or a laboratory accident (4); thus, smallpox is a high priority for preparedness planning (5). Given that smallpox is eradicated, mathematical models

Author affiliations: School of Public Health and Community Medicine, University of New South Wales, Sydney, New South Wales, Australia (C.R. MacIntyre, V. Costantino, X. Chen,

A.A. Chughtai, M. Kunasekaran); Arizona State University, Phoenix, Arizona, USA (C.R. Maclntyre); Monash University and Monash Health, Melbourne, Victoria, Australia (E. Segelov); Kirby Institute, University of New South Wales, Sydney (A. Kelleher); Emory University, Atlanta, Georgia, USA (J.M. Lane)

DOI: https://doi.org/10.3201/eid2404.171233 enable researchers to predict the effects of a smallpox epidemic, but these predictions depend critically on the assumptions of the mathematical model.

Many researchers who have developed smallpox models have been optimistic about residual vaccine-induced immunity and assumed a case-fatality ratio (CFR) of 30\%, whereas estimates of outbreaks in nonimmune populations suggest a CFR of $50 \%-70 \%$ (6). Given the absence of smallpox in the world for nearly 40 years and loss of immunologic boosting from wild-type infection, the CFR of an epidemic today might be higher.

The immunologic status of the population has also changed dramatically in the decades since smallpox eradication. A larger proportion of the population today is unvaccinated, and residual immunity in persons who were vaccinated before 1980 is waning (7). In addition, the prevalence of HIV, advances in transplantation, and therapies for cancer and many autoimmune conditions have resulted in unprecedented rates of immunosuppression (8). In 1980, when smallpox was eradicated, HIV had not yet manifested a high global burden of disease. Similarly, bone marrow transplantation was in its infancy, and heart-lung transplantations had not yet occurred. The fact that the proportion of unvaccinated and immunosuppressed persons in the population is increasing has not yet been adequately considered in estimations of the effect of reemergent smallpox.

Persons born after 1980 have no immunity to smallpox because they have never been exposed to wild-type virus or been vaccinated. For vaccinated cohorts, immunity wanes over time, and the highest protection is present during the first 5 years after vaccination, possibly waning to zero within 5-10 years (9). Furthermore, immunosenescence is a predictable, exponential decline in immune function that occurs after 50 years of age (10) and reduces the body's ability to fight infection and respond to vaccines (11). This phenomenon further adds to immunosuppression in countries with an aging population. The aim of this study was to estimate the effect of reemerging smallpox in New York, New York, USA, and Sydney, New South Wales, 
Australia, 2 large cities with different vaccination histories for which estimates could be made on the population's immunologic status.

\section{Methods}

\section{Population}

We used Sydney's population in 2015 (12), which was estimated using data from the state of New South Wales (13). The New York population of the same year was derived from the relevant statistical collection (14). We divided both populations into 5-year age groups up through ages $80-84$ years and combined the eldest (persons $>84$ years of age) into a single group (Figure 1, panel A). Each age group was divided into vaccinated and unvaccinated compartments, which were then further subdivided into 3 categories of immunity: immunocompetent, mildly immunosuppressed, and moderate-to-severely immunosuppressed. We assumed that immunosuppressed persons had no residual immunity from vaccination.

\section{Immunosuppressed Population}

We considered common types of immunosuppression estimated in an influenza study (15). We classified persons into 2 categories of immunosuppression: moderate to severe (called severe in our model) and mild. Severe immunosuppression was defined as a condition in which quantifiable data existed to demonstrate a risk for infection more than twice that of an immunocompetent person. This classification was left as a single category in the absence of reliable methodology to subdivide it. Mild immunosuppression was defined as a condition in which immunosuppression was documented but susceptibility to infection was estimated to be less than twice that of an immunocompetent host. For the analysis, persons with severe immunosuppression were assumed to have $2 \times$ and mild immunosuppression $1.5 \times$ the susceptibility to infection of a healthy person (16).

We sourced data for each city, and when only countrywide data were available, we attributed rates from the countrywide data set to the respective fraction of the population in the city. When age-specific immunosuppression prevalence data were not available, we used yearly

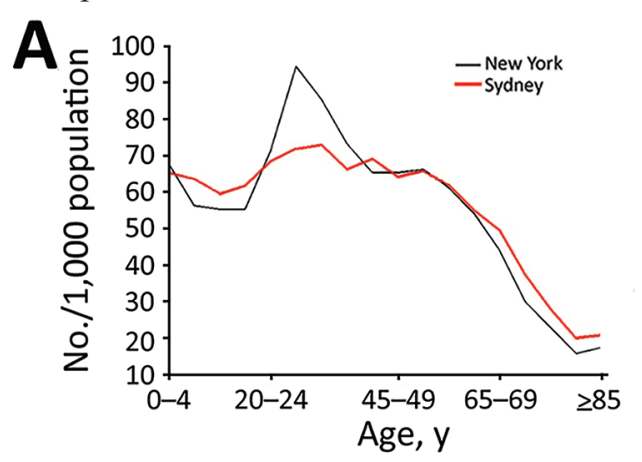

age-specific incidence data instead to calculate prevalence age distribution $(17,18)$.

We estimated the populations living with cancer $(17,19), \operatorname{HIV}(20,21)$, organ transplants $(22,23)$, respiratory syndromes such as asthma $(24,25)$ and chronic obstructive pulmonary disease $(26,27)$, dialysis $(28,29)$, and autoimmune diseases $(30,31)$ and divided these populations into the 2 immunosuppression categories for each city (online Technical Appendix Table 1, https://wwwnc.cdc.gov/EID/ article/24/4/17-1233-Techapp1.pdf). We acknowledge that many other diseases are associated with immunosuppression. Our method underestimates the amount of immunosuppression in the population.

\section{Vaccine-Induced Residual Immunity}

In the United States, including New York, widespread smallpox vaccination occurred until 1970 (32). In contrast, in the geographically isolated island continent of Australia, quarantine was used to protect the population because smallpox was never endemic (32). Widespread vaccination never occurred in Australia; only the armed forces and healthcare workers were vaccinated, which occurred until 1979, although reactive vaccination campaigns had been conducted during a smallpox outbreak in Sydney in 1917 (33).

For New York, we assumed $80 \%$ of the healthy population 40-69 years of age (born before 1975) were previously vaccinated. For Sydney, we estimated the proportion of persons vaccinated by estimating those born before 1980 in the following groups: healthcare workers in Sydney in 2015 (34), members of the defense forces, and migrants ( $>30 \%$ in the Sydney population) (35), who might have been vaccinated in their country of origin $(\approx 80,000$ persons). We estimated that, in Sydney, at most $30 \%$ of the total population born before 1980 (persons 35-69 years of age) had been vaccinated. On the basis of a mathematical model (36) that estimated waning immunity against severe smallpox as $1.41 \%$ per year after vaccination, we calculated the age-specific residual protection for vaccinated persons 40-69 years of age by multiplying that percentage by the number of years from vaccination and subtracting it from $100 \%$ starting protection.

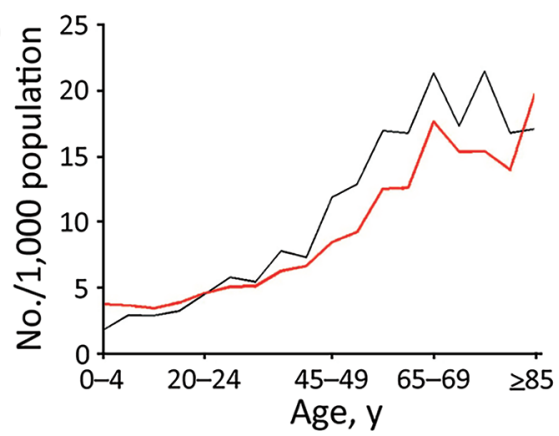

Figure 1. Characteristics of population used to model smallpox transmission, by age group, New York, NY, USA, and Sydney, New South Wales, Australia. Characteristics (e.g., size, age, immunosuppression rates) of populations from 2015 were used.

A) Total population;

B) immunosuppressed population. 


\section{Contact Mixing}

In our model, we used the heterogeneous age-specific contact rates from the European mixing patterns study (37). We assumed that persons would greatly reduce their social contacts after becoming symptomatic with smallpox (38). To account for this change in social contact, we modified the normal contact matrix, multiplying the matrix by a factor $(0<x<1)$ to reduce the number of contacts per day (39). Because of the lack of studies quantifying this reduction, we assumed $x$ to be 0.5 , as in a previous study (39). Considering severe smallpox types are more substantially prostrating, we applied the reduced contact matrix to hemorrhagic and flat smallpox infections from the first day of illness. For ordinary smallpox, we assumed the behavior change started on the second day and for vaccine-modified smallpox, on the third day.

\section{Disease Types}

We categorized smallpox disease into 4 different types defined by infectivity $\left(\mathrm{R}_{0}\right)$ and CFRs: hemorrhagic, flat, ordinary, and vaccine-modified. Age-specific and other model parameters (online Technical Appendix Table 2) as well as further model details are explained in the online Technical Appendix.

\section{Smallpox Disease Type Distribution}

We assumed infected persons had different probabilities of developing each disease type, depending on their age and immunologic status. The incidence of each disease type within each age group for healthy unvaccinated persons was drawn from historical outbreaks (9) (online Technical Appendix Table 3). For healthy unvaccinated persons, hemorrhagic smallpox ratios ranged from 7 cases/1,000 persons infected (in the 5-9-year age group) to 200 cases/1,000 persons infected (in the $\geq 85$-year age group). Flat smallpox age-specific rates were lowest for the 10-14-year age group (30 cases/1,000 persons infected) and reached 180 cases/1,000 persons infected for the oldest age group. For the mildly immunocompromised population, we doubled the age-specific probability of hemorrhagic and flat smallpox. We assumed $100 \%$ of severely immunocompromised persons would develop hemorrhagic disease. We assumed the vaccinated subgroup had reduced susceptibility and rates of severe smallpox types. We estimated that $25.3 \%$ of vaccinated persons would get vaccine-modified smallpox (9). We applied a waning immunity function over time at a rate of $1.41 \%$ per year from vaccination (36) and assumed the rates of hemorrhagic and flat smallpox would increase with time from vaccination while rates of vaccinemodified smallpox would decrease with time from vaccination (online Technical Appendix Table 4).

\section{Mathematical Model}

We constructed a modified SEIR (susceptible, exposed, infected, recovered) model for smallpox transmission (online
Technical Appendix Table 2). The population was divided into vaccinated and unvaccinated compartments, which were then further subdivided into 3 categories of immunity: immunocompetent, mildly immunosuppressed, and moderate-to-severely immunosuppressed. The model used ordinary differential equations to move populations into epidemiologic states related to their smallpox infectious status: susceptible, infected, prodromal, infectious, recovered, or dead. Once infected, populations were moved into the next state on the basis of disease duration rates. To obtain the age-specific force of infection (i.e., the rate at which susceptible persons acquire smallpox), we used the Euler approximation to make discrete contact rates, assuming the rates were proportional to the patterns observed in the United Kingdom. Then, to account for the different infectivity rates of different smallpox types, we estimated the transmission parameter $\beta$ (i.e., the probability of getting infected from a contact) for each smallpox disease type to calculate the $\mathrm{R}_{0}$ for hemorrhagic, flat, ordinary, and vaccine-modified smallpox. Finally, we multiplied the force of infection by a parameter $\left(\alpha_{1}, \alpha_{2}, \alpha_{3}, \alpha_{4}\right.$; online Technical Appendix Table 2 ) to account for the different susceptibility levels of different populations.

The model ran for 100 simulated days. We assumed an attack in a crowded public space, such as an airport, and started the epidemic with 51 infected in New York and 29 in Sydney to reflect the same attack rate for each population. We assumed a dynamic population updated each day using the birth $(40)$ and age-specific death rates $(41,42)$ from 2014 for both cities.

\section{Sensitivity Analysis}

We conducted a sensitivity analysis on the assumption of waning immunity, reducing immunity by $0.7 \%$ per year (approximately half the value used in the base case scenario [i.e., the first scenario discussed]). We present results for 3 different assumptions about residual vaccine immunity: no residual immunity, base case immunity (1.41\% waning immunity per year), and high residual immunity (0.7\% waning immunity per year). We also conducted a sensitivity analysis to test the model outputs without considering population immunosuppression, which has been the approach in most past models (43).

\section{Results}

\section{Population and Immunity Levels}

We examined the population age distributions of New York and Sydney. Sydney has a higher percentage of persons $<20$ and $>55$ years of age than New York (Figure 1, panel A), whereas New York has a higher proportion of persons 20-39 years of age than Sydney. We estimated that $4.54 \%$ of New York's population and $3.76 \%$ 
of Sydney's population are severely immunocompromised, $14.81 \%$ of New York's population and 12.95\% of Sydney's population are mildly immunocompromised, $59.14 \%$ of New York's population and $72.56 \%$ of Sydney's population are healthy unvaccinated, and $21.51 \%$ of New York's population and $10.73 \%$ of Sydney's population are healthy vaccinated. Similar proportions of the 2 cities' populations (19\% in New York and $17 \%$ in Sydney) are immunosuppressed (Figure 1, panel B). New York has a higher proportion of the population vaccinated $(21 \%)$ than Sydney (10\%).

\section{Base Case Scenario}

We analyzed age-specific infection (Figure 2, panel A) and death (Figure 2, panel B) rates using the base case scenario (medium immunity level) including the immunosuppressed population. Persons 5-19 years of age are at highest risk for smallpox infection in both cities (Figure 2, panel A). Although the proportion of persons infected in both cities is similar among the $0-19$-year age groups, $\approx 25 \%$ more persons in New York than Sydney become infected among the 20-39-year age groups.

Cumulative deaths per 1,000 population increase with age starting with persons $\geq 20$ years of age (Figure 2, panel B). Deaths peak in the 65-69-year age group in both cities, reaching 1.2 deaths $/ 1,000$ population for New York and 0.9 deaths/1,000 population for Sydney 60 days after the start of the outbreak; rates increase again in those $\geq 80$ years of age. The New York population also has a smaller peak in deaths in the 35-39-year age group. Although the spread of infection is mostly driven by higher contact rates among persons of young age groups, the peaks in death reflect the distribution of the immunosuppressed population (Figure 1, panel B; Figure 2, panel B). The effect of residual immunity is more apparent in New York trends, which show a greater decrease in infections and cumulative deaths in the age groups that were previously vaccinated (40-65 years of age).

Looking at total rates over time, New York (Figure 3, panels A, C) and Sydney (Figure 3, panels B, D) have similar exponential growths of infection rates, with slightly higher trends for New York. The rate of infection reaches 0.094 infected/1,000 population for New York and 0.084 infected/1,000 population for Sydney 50 days after the smallpox introduction and increases to 0.496 infected/1,000 population for New York and 0.452 infected/1,000 population for Sydney by 70 days. The death rates are 0.028 deaths $/ 1,000$ population for New York and 0.025 deaths $/ 1,000$ population for Sydney after 50 days and reach 0.151 deaths $/ 1,000$ population for New York and 0.133 deaths $/ 1,000$ population for Sydney by 70 days.

\section{Residual Immunity Analysis}

Infection and death rate estimates for New York, where vaccine coverage is more than double that of Sydney, are more sensitive to assumptions of residual immunity. New York (Figure 3, panel A) has lower rates of infection than Sydney (Figure 3, panel B) only in the scenario of high residual immunity. At day 50 of the outbreak, rates are $\approx 15 \%$ (base case residual immunity) and 31\% (high residual immunity) lower in New York and 10\% (base case residual immunity) and $17 \%$ (high residual immunity) lower in Sydney with residual immunity than without residual immunity. Differences in infection and death rates among different residual immunity assumptions increased with time. Regarding the impact on age-specific rates in New York (Figure 4, panel A) and Sydney (Figure 4, panel B), the assumption of high residual immunity produced lower death rates for the older age groups.

\section{Immunosuppression Analysis}

Infection and death rates increase when including (vs. excluding) immunosuppression parameters in the model; greater differences are seen between New York's infection rates (Figure 3, panel C) and Sydney's infection rates (Figure 3, panel D). The difference in rates increases with time, reaching $\approx 20 \%$ in New York and $18 \%$ in Sydney at day 50 from the start of the outbreak and 28\% for New York and $25 \%$ for Sydney at day 70 . Although including immunosuppression estimates into the model produced similarly higher infection rates for each age group (less for the 0-4year age group), differences in death rates increased with age (Figure 4).
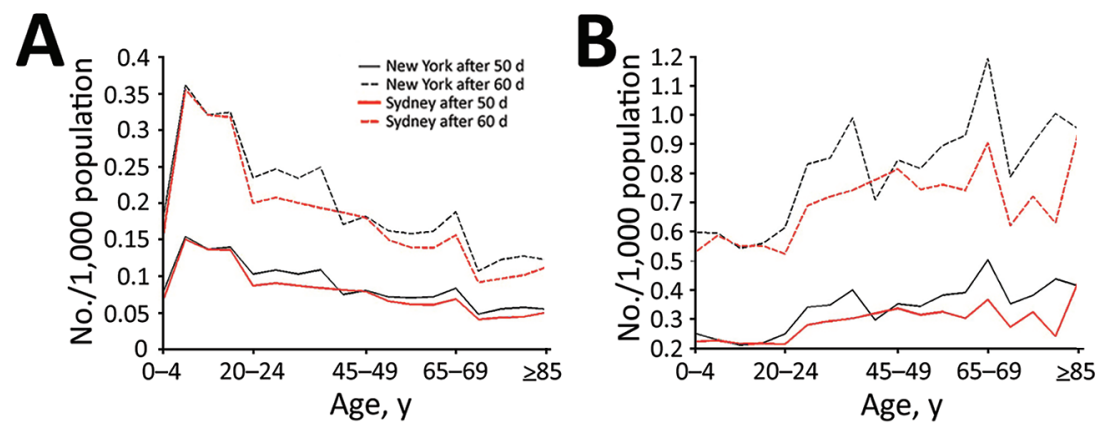

Figure 2. Smallpox infection and death rates of population for base case scenario and for scenario including immunosuppression in model, by age group, New York, NY, USA, and Sydney, New South Wales, Australia. Characteristics (e.g., size, age, immunosuppression rates) of populations from 2015 were used. A) Infection rate 50 and 60 days after start of smallpox outbreak; B) cumulative deaths in population 50 and 60 days after start of smallpox outbreak. 

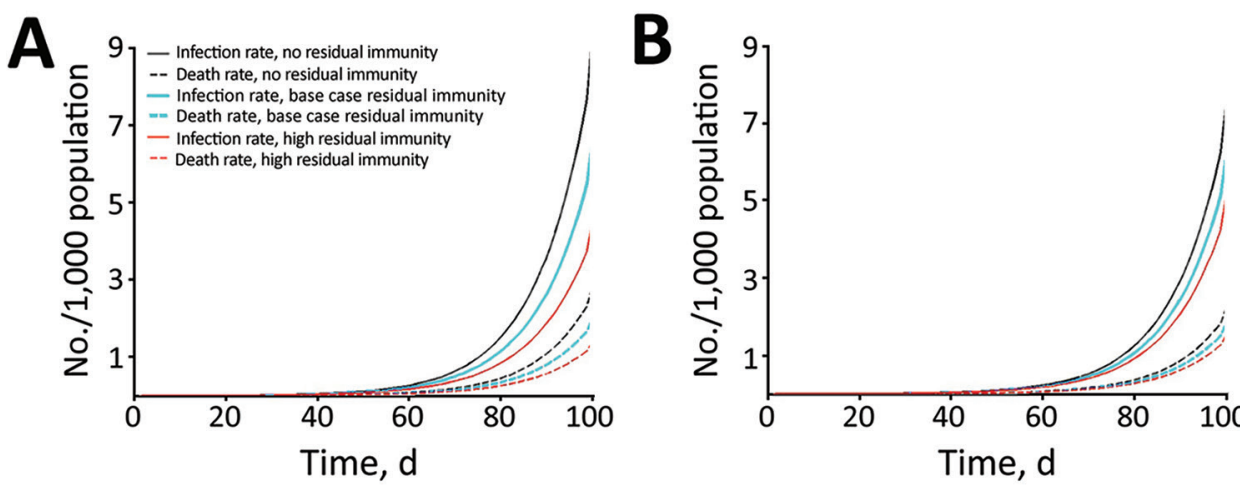

C

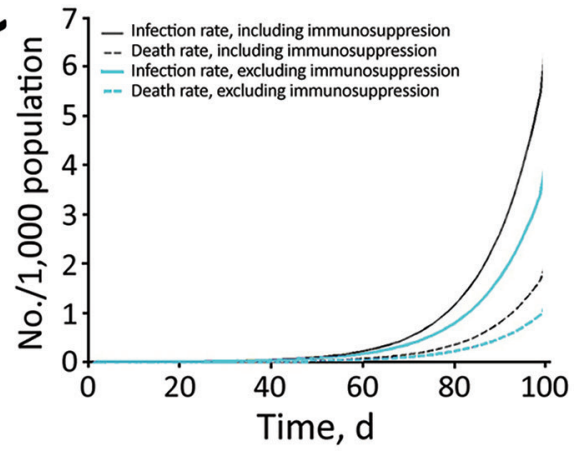

D

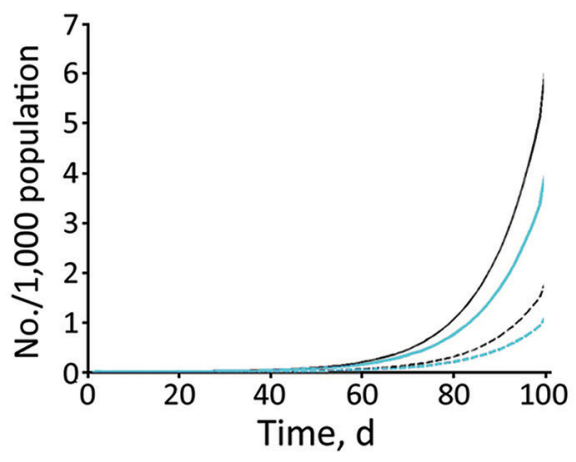

Figure 3. Smallpox infection and death rates over time considering different immunologic factors included in model, New York, NY, USA, and Sydney, Australia. Characteristics (e.g., size, age, immunosuppression rates) of populations from 2015 were used. A) Rates for New York, considering different levels (none, base case, and high) of residual vaccine immunity with the inclusion of immunosuppressed population.

B) Rates for Sydney, considering different levels (none, base case, and high) of residual vaccine immunity with the inclusion of immunosuppressed population. C) Rates for New York, including and excluding immunosuppression with base case level of residual vaccine immunity. D) Rates for Sydney, including and excluding immunosuppression with

base case level of residual vaccine immunity.

\section{Discussion}

With each passing year, population immunosuppression is a more influential determinant than residual vaccine immunity of the severity of a smallpox epidemic. Although the spread of disease is highest in younger age groups, driven mostly by their higher contact rates, higher death rates were seen in older populations, reflecting the prevalence of immunosuppression.

The differences between New York, which has high vaccination coverage (an estimated $\approx 22 \%$ of the population), and Sydney, which has low $(\approx 10 \%)$ vaccination coverage, demonstrate that residual immunity assumptions are not as influential in Sydney as in New York. However, the consideration of population immunosuppression, from medical conditions to iatrogenic factors, strongly affects disease transmission and deaths in both cities. This large population subset must be considered when modeling the impact of any infectious disease outbreak. We estimated conservatively that almost 1 in 5 persons in New York and 1 in 6 persons in Sydney (and higher for the 60-64-year age group) are living with some degree of immunosuppression. Although New York has higher rates of immunosuppression for the 25-84-year age groups, Sydney has higher rates than New York for the youngest (0-19 years) and the oldest $(\geq 85)$ populations.

Residual immunity affects age-specific infection and death rates, with both cities showing the highest infection rates for unvaccinated young persons 5-19 years of age. However, death rates rise after 40 years of age, despite higher vaccination coverage in this age group. For Sydney, even an assumption of higher immunity does not affect the infection or death rates greatly because of the low vaccine coverage before 1980 . However, residual immunity becomes more influential if we use more optimistic assumptions of waning immunity. Note that persons who have been vaccinated would mount a more robust and rapid response to revaccination in the event of an outbreak and might be better protected after postexposure vaccination. Obtaining a vaccination history and checking for a consistent scar are necessary parts of outbreak management.

Although immunosuppression is a major determinant of the size and distribution of a smallpox outbreak, this fact should not be a major determinant of vaccination policy. Immunosuppression should continue to be an absolute contraindication for vaccination of persons who are not true contacts. Ensuring that persons with immunosuppression (including healthcare workers) avoid contact with persons with smallpox (if possible) should be a priority. Smallpox would always be more pathogenic than vaccinia virus, so any patient with a bona fide exposure to smallpox should be vaccinated with a fully potent vaccinia strain, such as ACAM2000 (44). If such patient develops a serious complication, such as eczema vaccinatum or progressive vaccinia, the patient can be treated with ST-246 (Siga Technologies, New York, NY, USA) (45). 

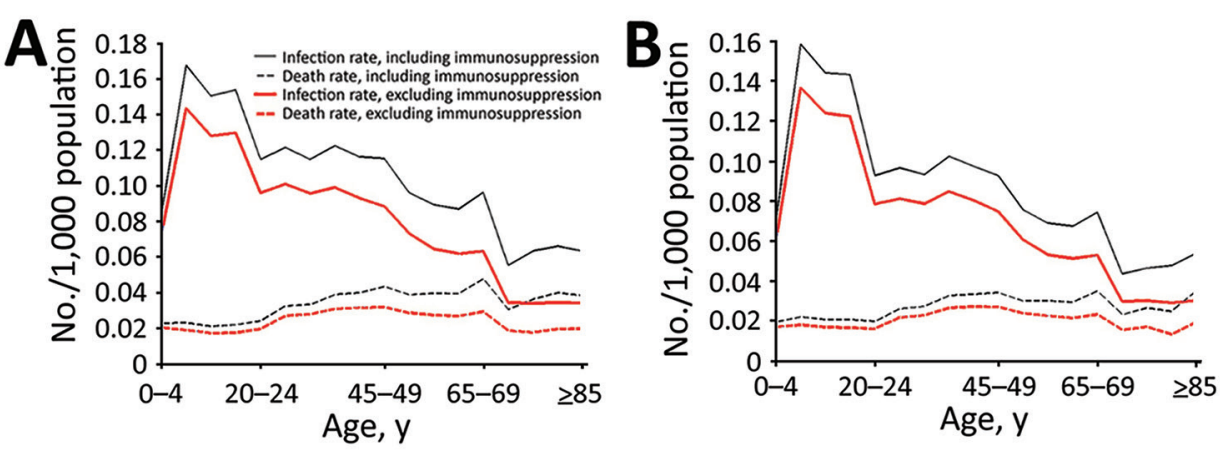

Figure 4. Smallpox infection and death rates with different levels of residual vaccine immunity including and excluding immunosuppression in model of smallpox transmission, by age group, New York, NY, USA, and Sydney, Australia. Characteristics (e.g., size, age, immunosuppression rates) of populations from 2015 were used. A) New York 50 days after start of smallpox outbreak with no (top), base case (middle), and
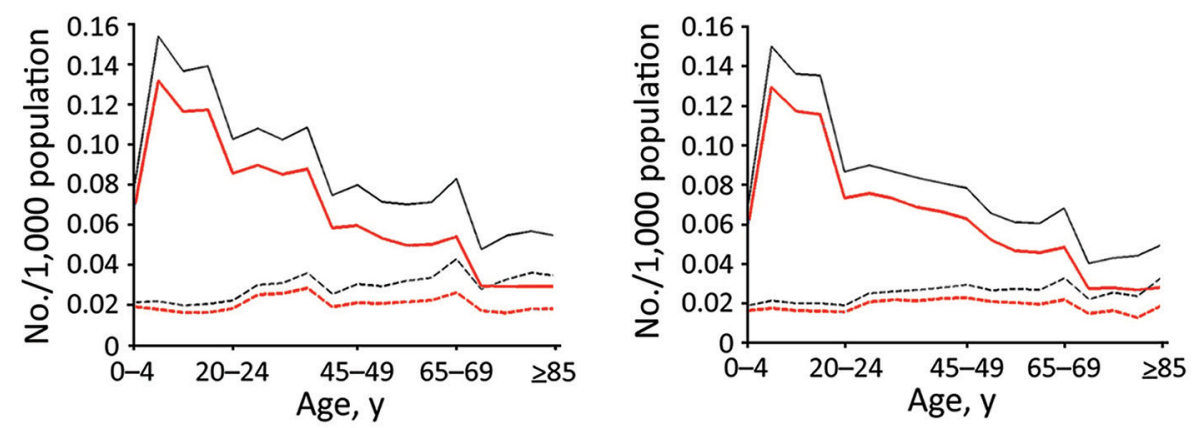
high (bottom) residual vaccine immunity. B) Sydney 50 days after start of smallpox outbreak with no (top), base case (middle), and high (bottom) residual vaccine immunity.
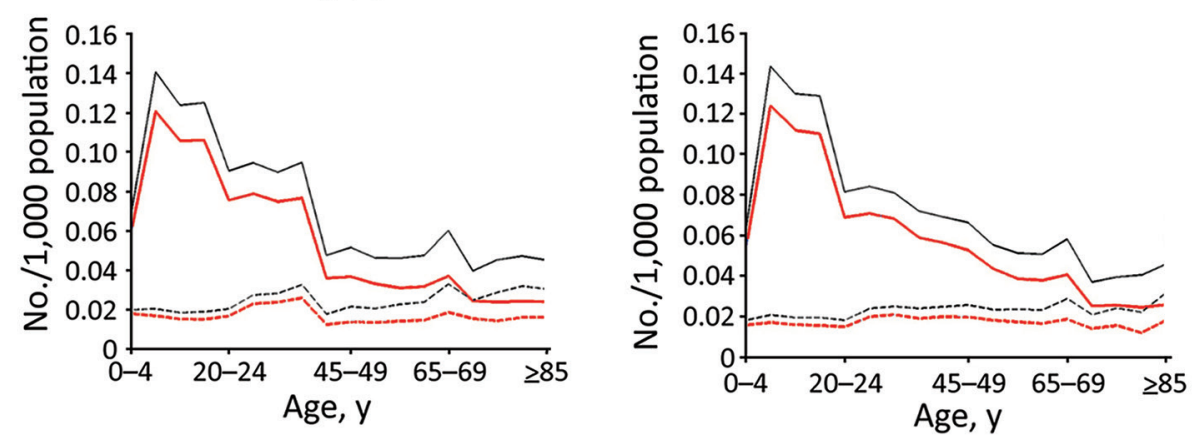

Our study is subject to some limitations. We used an underestimate of immunosuppression; other conditions causing immunosuppression, such as diabetes, were not considered. We also used conservative estimates for the increased risk for infection in immunosuppressed persons and grouped persons with severe and moderate immunosuppression into single categories because of the absence of more specific data to categorize them further by degree of immunosuppression. The contact matrix we used was estimated in a study conducted in the United Kingdom in 2006, which might not necessarily reflect New York or Sydney social contact patterns (37). Furthermore, contacts with symptomatic infectious patients will probably drop to near zero once an outbreak has been confirmed and patients are well isolated, assuming adequate health system capacity for isolation and treatment of smallpox patients. The data in the model on age-specific rates of smallpox were obtained from hospitalized patients (9), which might overestimate the rates of severe disease in the model outputs.

The speed and vigor with which smallpox control efforts are implemented should be major aspects of control efforts and need to be tested in a model that accounts adequately for immunosuppression. Ensuring adequate hospital care and isolation facilities will also help in epidemic control. During the Ebola epidemic in West Africa, lack of beds resulted in widespread community transmission, and modeling showed that $70 \%$ of patients needed to be in treatment facilities to control the epidemic (46). The response to severe acute respiratory syndrome, with its rapid control despite the lack of a vaccine or antiviral agent, showed that patient isolation can be very successful (47). Experiences with severe acute respiratory syndrome, Ebola, and Middle East respiratory syncytial coronavirus also illustrate the heavy toll on healthcare workers (48), who should be assumed to be at high risk for infection in the event of a smallpox outbreak.

Given waning smallpox vaccine immunity (nearly 4 decades since eradication and a dwindling vaccinated population), the influence of population immunosuppression is 
greater than that of residual vaccine immunity, yet has not been adequately considered in smallpox epidemic modeling. Advances in medicine and new endemic diseases, such as HIV, have resulted in almost 1 in 5 persons living with immunosuppression in large metropolitan cities. Immunosuppression must be considered in preparedness planning and poses a challenge for vaccination strategies during potential smallpox outbreaks.

\section{About the Author}

Dr. MacIntyre is professor of Infectious Diseases Epidemiology at the University of New South Wales, Sydney, and leads a research program on biosecurity, bioterrorism, and emerging infectious diseases, which are her primary research interests.

\section{References}

1. Centers for Disease Control and Prevention. General fact sheets on specific bioterrorism agents. 2017 May 9 [cited 2017 Jan 24]. https:/emergency.cdc.gov/bioterrorism/factsheets.asp

2. Fenner F, Henderson DA, Arita I, Ježek Z, Ladnyi ID. Smallpox and its eradication. Geneva: World Health Organization; 1987.

3. Koblentz GD. The de novo synthesis of horsepox virus: implications for biosecurity and recommendations for preventing the reemergence of smallpox. Health Secur. 2017;15:620-8. http://dx.doi.org/10.1089/hs.2017.0061

4. MacIntyre CR. Biopreparedness in the age of genetically engineered pathogens and open access science: an urgent need for a paradigm shift. Mil Med. 2015;180:943-9. http://dx.doi.org/ 10.7205/MILMED-D-14-00482

5. MacIntyre CR, Seccull A, Lane JM, Plant A. Development of a risk-priority score for category A bioterrorism agents as an aid for public health policy. Mil Med. 2006;171:589-94. http://dx.doi.org/ 10.7205/MILMED.171.7.589

6. Gelfand HM, Posch J. The recent outbreak of smallpox in Meschede, West Germany. Am J Epidemiol. 1971;93:234-7. http://dx.doi.org/10.1093/oxfordjournals.aje.a121251

7. World Health Organization. Smallpox [cited 2017 Feb 28]. http://www.who.int/csr/disease/smallpox/en/

8. Parrino J, Graham BS. Smallpox vaccines: past, present, and future. J Allergy Clin Immunol. 2006;118:1320-6. http://dx.doi.org/10.1016/j.jaci.2006.09.037

9. Rao AR. Smallpox. Bombay (India): Kothari Book Depot; 1972.

10. Montecino-Rodriguez E, Berent-Maoz B, Dorshkind K. Causes, consequences, and reversal of immune system aging. J Clin Invest. 2013;123:958-65. http://dx.doi.org/10.1172/JCI64096

11. Raina MacIntyre C, Menzies R, Kpozehouen E, Chapman M, Travaglia J, Woodward M, et al. Equity in disease prevention: vaccines for the older adults - a national workshop, Australia 2014. Vaccine. 2016;34:5463-9. http://dx.doi.org/10.1016/ j.vaccine.2016.09.039

12. Australian Bureau of Statistics. Australian demographic statistics. 2017 [cited $2018 \mathrm{Feb} 8$ ]. http://www.ausstats.abs.gov.au/ausstats/ subscriber.nsf/0/0DEC5B368C5C2D72CA2581F5001011EB/ \$File/31010 jun 2017.pdf

13. Australian Bureau of Statistics. Australian historical population statistics, 2014. 2014 Sep 18 [cited 2017 Mar 14]. http://www.abs.gov.au/AUSSTATS/abs@.nsf/mf/3105.0.65.001

14. Baruch College. New York City (NYC) age and sex distributionby county. 2015 [cited 2017 Mar 17]. http://www.baruch.cuny.edu/ nycdata/population-geography/age_distribution.htm\#
15. Kunisaki KM, Janoff EN. Influenza in immunosuppressed populations: a review of infection frequency, morbidity, mortality, and vaccine responses. Lancet Infect Dis. 2009;9:493-504. http://dx.doi.org/10.1016/S1473-3099(09)70175-6

16. Finin P, Kosaraju A, Rose E, Rubin H. The role of vaccination, antiorthopoxvirus drug, and social cooperativity in a mathematical model of smallpox control. Biosecur Bioterror. 2013;11:59-72. http://dx.doi.org/10.1089/bsp.2012.0037

17. Australian Government. Cancer in Australia statistics. 2017 [cited 2017 Feb 15]. https://canceraustralia.gov.au/affected-cancer/ what-cancer/cancer-australia-statistics

18. New York State. Cancer incidence and mortality by age group, New York State, 2010-2014. 2016 [cited 2017 Mar 17]. https://www.health.ny.gov/statistics/cancer/registry/table6/ tb6totalnys.htm

19. New York State, Department of Health. NYS cancer registry and cancer statistics. 2017 [cited 2018 Feb 8]. https://www.health.ny.gov/ statistics/cancer/registry/

20. New South Wales Ministry of Health. NSW HIV strategy 2012-2015. 2015 [cited 2017 Feb 15]. http://www.health.nsw.gov. au/endinghiv/Documents/q4-2015-and-annual-hiv-data-report.pdf

21. Centers for Disease Control and Prevention. Diagnoses of HIV infection in the United States and dependent areas, 2015. HIV Surv Rep. 2015;27 [cited 2017 Mar 22]. https://www.cdc.gov/ hiv/pdf/library/reports/surveillance/cdc-hiv-surveillance-report2015-vol-27.pdf

22. New South Wales Ministry of Health. Increasing organ donation in NSW, government plan 2012. 2012 Aug [cited 2017 Feb 15]. http://www.health.nsw.gov.au/organdonation/Publications/ increasing-organ-donation.pdf

23. United Network for Organ Sharing. Annual reports. A look back at UNOS' achievements. 2015 [cited 2017 Mar 16]. https://www.unos.org/about/annual-report/

24. Australian Institute of Health and Welfare. Who gets asthma? 2016 [cited 2017 Feb 15]. https://www.aihw.gov.au/reports/asthma-otherchronic-respiratory-conditions/asthma/contents/who-gets-asthma/

25. Centers for Disease Control and Prevention. Most recent asthma data. 2017 [cited 2017 Mar 16]. https://www.cdc.gov/asthma/ most recent data.htm

26. Australian Institute of Health and Welfare. Chronic diseases data. 2016 [cited 2018 Feb 8]. https://www.aihw.gov.au/reports/ life-expectancy-death/grim-books/contents/grim-books\#page2

27. Centers for Disease Control and Prevention. Chronic obstructive pulmonary disease. COPD among adults in New York. 2012 [cited 2017 Mar 17]. https://www.cdc.gov/copd/maps/docs/pdf/ NY_COPDFactSheet.pdf

28. McDonald S, Chang S, Excell L, editors. Australia and New Zealand dialysis and transplant registry. Adelaide (South Australia, Australia): The Queen Elizabeth Hospital; 2008.

29. Dialysis Patient Citizens. New York. 2015 [cited 2017 Mar 22]. http://www.dialysispatients.org/advocacy/state-resources/new-york

30. Australasian Society of Clinical Immunology and Allergy. Autoimmune diseases. 2017 [cited 2017 Mar 24]. https://www. allergy.org.au/patients/autoimmunity/autoimmune-diseases

31. Cooper GS, Bynum ML, Somers EC. Recent insights in the epidemiology of autoimmune diseases: improved prevalence estimates and understanding of clustering of diseases. J Autoimmun. 2009;33:197-207. http://dx.doi.org/10.1016/j.jaut.2009.09.008

32. Kennedy RB, Lane JM, Henderson DA, Poland GA. Smallpox and vaccinia. In: Plotkin SA, Orenstein WA, Offit PA, editors. Vaccines, 6th ed. Amsterdam: Elsevier Inc.; 2012. p. 718-45.

33. Australian Government Department of Health. Guidelines for smallpox outbreak, preparedness, response and management. 2004 [cited 2017 Apr 4]. http://www.health.gov.au/internet/main/ publishing.nsf/content/health-pubhlth-publicat-documentmetadata-smallpox.htm 
34. Australian Institute of Health and Welfare. Australia's medical workforce 2012. 2014 Jan 24 [cited 2017 Apr 4]. https://www.aihw.gov. au/reports/workforce/medical-workforce-2012/contents/summary

35. Australian Bureau of Statistics. Migration, Australia, 2009-10. 2011 Jun 16 [cited 2017 Aug 3]. http://www.abs.gov.au/ AUSSTATS/abs@.nsf/DetailsPage/3412.02009-10?OpenDocument

36. Eichner M. Analysis of historical data suggests long-lasting protective effects of smallpox vaccination. Am J Epidemiol. 2003;158:717-23. http://dx.doi.org/10.1093/aje/kwg225

37. Mossong J, Hens N, Jit M, Beutels P, Auranen K, Mikolajczyk R, et al. Social contacts and mixing patterns relevant to the spread of infectious diseases. PLoS Med. 2008;5:e74. http://dx.doi.org/ 10.1371/journal.pmed.0050074

38. Van Kerckhove K, Hens N, Edmunds WJ, Eames KTD. The impact of illness on social networks: implications for transmission and control of influenza. Am J Epidemiol. 2013;178:1655-62. http://dx.doi.org/10.1093/aje/kwt196

39. Del Valle SY, Hyman JM, Chitnis N. Mathematical models of contact patterns between age groups for predicting the spread of infectious diseases. Math Biosci Eng. 2013;10:1475-97. http://dx.doi.org/10.3934/mbe.2013.10.1475

40. Mundi I. Historical data graphs per year. 2015 Jun 30 [cited 2017 May 8]. http://www.indexmundi.com/g/g.aspx? $\mathrm{v}=25 \& \mathrm{c}=\mathrm{as} \& \mathrm{l}=\mathrm{en}$

41. Australian Bureau of Statistics. Deaths, year of occurrence, age at death, age-specific death rates, sex, states, territories and Australia. 2017 [cited 2017 May 8]. http://stat.data.abs.gov.au/ Index.aspx? Queryid=457

42. Kochanek KD, Murphy SL, Xu J, Tejada-Vera B. Deaths: Final Data for 2014. Natl Vital Stat Rep. 2016;65:1-122.
43. Ren Y, Ordóñez F, Wu S. Optimal resource allocation response to a smallpox outbreak. Comput Ind Eng. 2013;66:325-37. http://dx.doi.org/10.1016/j.cie.2013.07.002

44. Berhanu A, King DS, Mosier S, Jordan R, Jones KF, Hruby DE, et al. Impact of ST-246® on ACAM $2000^{\mathrm{TM}}$ smallpox vaccine reactogenicity, immunogenicity, and protective efficacy in immunodeficient mice. Vaccine. 2010;29:289-303. http://dx.doi.org/10.1016/j.vaccine.2010.10.039

45. Grosenbach DW, Jordan R, Hruby DE. Development of the small-molecule antiviral ST-246 as a smallpox therapeutic. Future Virol. 2011;6:653-71. http://dx.doi.org/10.2217/fvl.11.27

46. Meltzer MI, Atkins CY, Santibanez S, Knust B, Petersen BW, Ervin ED, et al.; Centers for Disease Control and Prevention. Estimating the future number of cases in the Ebola epidemic Liberia and Sierra Leone, 2014-2015. MMWR Suppl. 2014; 63:1-14.

47. Guan Y, Zheng BJ, He YQ, Liu XL, Zhuang ZX, Cheung CL, et al. Isolation and characterization of viruses related to the SARS coronavirus from animals in southern China. Science. 2003;302:276-8. http://dx.doi.org/10.1126/ science. 1087139

48. Heymann DL. Smallpox containment updated: considerations for the 21st century. Int J Infect Dis. 2004;8(Suppl 2):S15-20. http://dx.doi.org/10.1016/j.ijid.2004.09.005

Address for correspondence: Valentina Costantino, University of New South Wales Faculty of Science, SPHCM, Kensington Gate 9, Sydney, New South Wales 2052, Australia; email: v.costantino@unsw.edu.au

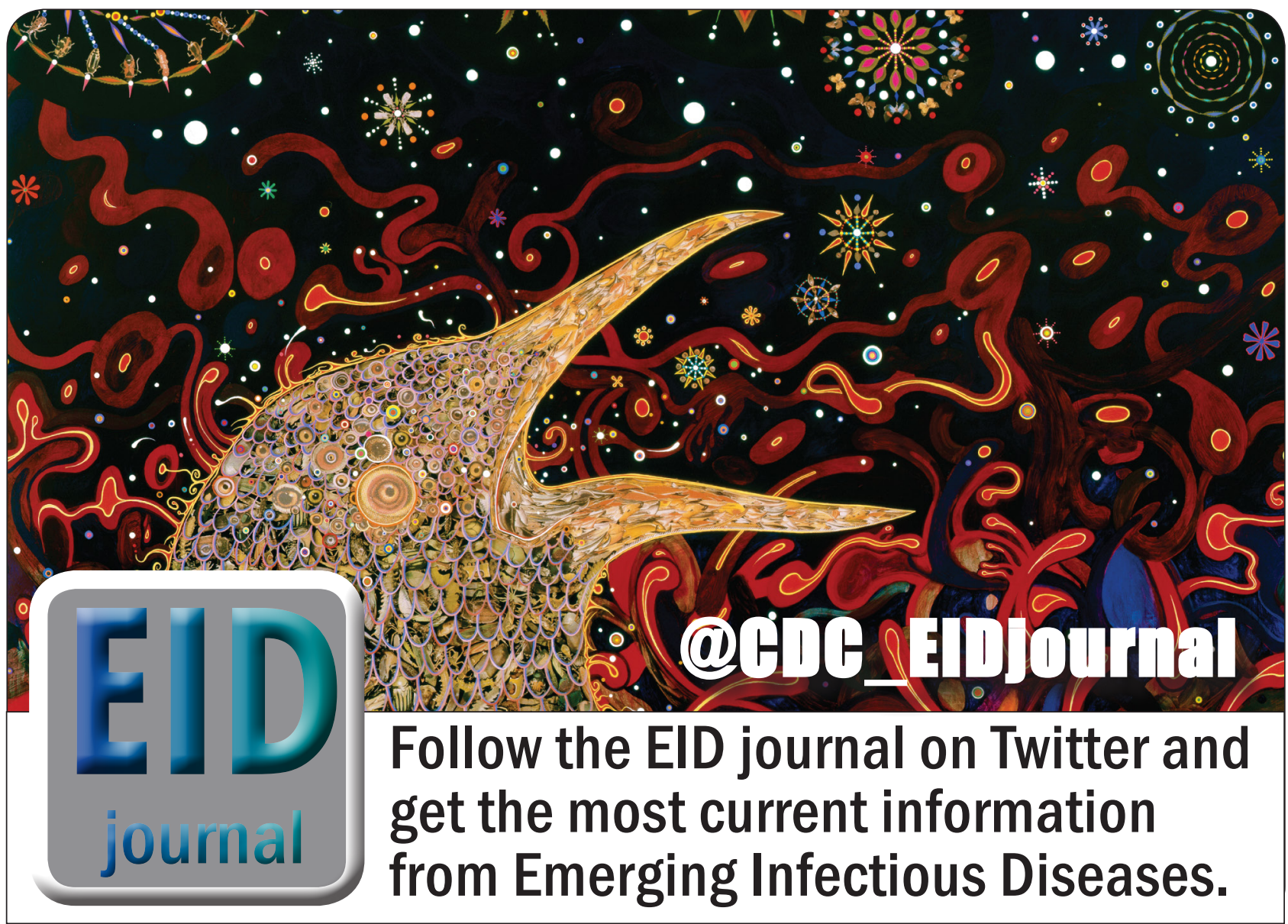

